\title{
Lesões no Colo Uterino sob Visão Colposcópica: Achados Anatomopatológicos em Serviço Ginecológico
}

\author{
Cervical Lesions UnderColposcopic Vision: Anatomopathological Findings in \\ Gynecological Service
}

Lesiones en el Colo Uterino bajo Visión Colposcópica: Hallazgos Anatomopatológicos en Servicio Ginecológico.

José Humberto Belmino Chaves ${ }^{1}$

Alessandra Plácido Lima Leite ${ }^{2}$

Antônio Fernando de Sousa Bezerra ${ }^{3}$

Márcio Felipe Soares Sales ${ }^{4}$

Rayana Valéria da Cruz Neves ${ }^{5}$

\section{Resumo}

Objetivo: Avaliar o anatomopatológico obtido através da classificação colposcópica da zona de transformação e o resultado da colposcopia, citologia e biópsia prévios e em seguimento por $6 \mathrm{e}$
12 meses após o tratamento. Método: Trata-se de um estudo epidemiológico, transversal e retrospectivo, com amostra obtida através de prontuários e laudos de anatomopatológico de pacientes mulheres maiores de 18 anos, atendidas no ambulatório de Patologias do Trato

${ }^{1}$ Médico. Graduado pela UFAL. Docente da Disciplina de Ginecologia da FAMED /UFAL. Doutor em Comunicação em Saúde pela Universidade Aberta de Lisboa-Portugal e em Bioética e Ética Médica pela Faculdade de Medicina da Universidade do Porto-Portugal.Autor correspondente: Campus A.C. Simões Av. Lourival Melo Mota, s/n . Tabuleiro do Martins. CEP: 57072-900. Maceió, Al, Brasil. E-mail: jhbchaves@uol.com.br

${ }^{2}$ Médica. Graduada pela UNCISAL. Docente da Disciplina de Ginecologia da FAMED/UFAL. DoutoraemCiências pela Universidade Federal de São Paulo.

${ }^{3}$ Médico. Graduado pela FAMED/UFAL. Docente da Disciplina de Patologia da FAMED /UFAL. Doutor em Patologia pelo Hospital Universitário Antônio Pedro pela Universidade Federal Fluminense.

${ }^{4}$ Enfermeira. Docente da UNCISAL.

${ }^{5,6}$ Graduando(a) no curso de Medicina pela FAMED /UFAL.

Recebido: Ago./2018 - Aceito: Dez./2018. 
Genital Inferior de um hospital universitário no período compreendido entre janeiro de 2012 a dezembro de 2016, que realizaram conização para tratamento de lesão de alto grau de colo uterino e que possuam dados de citologia, colposcopia, biópsia e anatomopatológico descritas no prontuário. Resultados: Os resultados encontrados nessa pesquisa, apresentam concordância com outros estudos realizados na literatura. A amostra compreende-se mulheres portadoras de Lesão Intraepitelial de Alto Grau, na sua maioria entre a faixa etária de 30 e 40 anos. Depreende-se também, alta associação entre a multiparidade com as lesões de alto grau e baixa significância na discordância entre os métodos diagnósticos avaliados. Conclusão: A detecção e o tratamento adequado das lesões precursoras é a base para o controle da doença. Sendo assim, são necessários esforços para melhorar a sensibilidade e especificidade testes diagnósticos, assim como para aumentar a conscientização das mulheres sobre os fatores de exposição ao HPV e a necessidade de adesão aos programas de controle.

\section{Descritores: Neoplasia;}

Papillomaviridae; Terapêutica.

\section{Abstract}

Objective: To evaluate the pathology obtained through the colposcopic classification of the transformation zone and the results of prior colposcopy, cytology and biopsy, followed for 6 and 12 months after treatment. Method: This is a cross-sectional and retrospective epidemiological study, with a sample obtained from medical records and reports of patients under 18 years of age, treated at the Outpatient Clinic of Lower Genital Tract of universitary hospital in the period between January 2012 to December 2016, who underwent conization for the treatment of high grade cervix lesions and have data on cytology, colposcopy, biopsy and pathology described in the medical record. Results: The results found in this study are consistent with other studies in the literature. The sample comprised women with High-Grade Intraepithelial Lesion, most of them between the ages of 30 and 40 years. It is also possible to show a high association between multiparity and high-grade lesions and low significance in the discordance between the diagnostic methods evaluated. Conclusion: The detection and appropriate treatment of precursor lesions is the basis for disease control. 
Thus, efforts are needed to improve sensitivity and specificity of diagnostic tests, as well as to increase women's awareness of HPV exposure factors and the need to adhere to control programs.

\section{Descriptors:}

\section{Neoplasms;}

Papillomaviridae; Therapeutics.

\section{Resumen}

Objetivo:

Evaluar

el

anatomopatológico obtenido a través de la clasificación colposcópica de la zona de transformación y el resultado de la colposcopia, citología y biopsia previas y en seguimiento por 6 y 12 meses después del tratamiento. Método: Se trata de un estudio epidemiológico, transversal y retrospectivo, con muestra obtenida a través de prontuarios y laudos de anatomopatológico de pacientes mujeres mayores de 18 años, atendidas en el ambulatorio de Patologías del Trato Genital Inferior del hospital Universitario en el periodo comprendido entre enero de 2012 a diciembre de 2016, que realizaron conización para tratamiento de lesión de alto grado de cuello uterino y que poseen datos de citología, colposcopia, biopsia y anatomopatológico descritas en el prontuario. Resultados: Los resultados encontrados en esta investigación, presentan concordancia con otros estudios realizados en la literatura. La muestra comprende mujeres portadoras de Lesión Intraepitelial de Alto Grado, en su mayoría entre el grupo de edad de 30 y 40 años. Se desprende también, alta asociación entre la multiparidad con las lesiones de alto grado y baja significancia en la discordancia entre los métodos diagnósticos evaluados. Conclusión: La detección y el tratamiento adecuado de las lesiones precursoras es la base para el control de la enfermedad. Por lo tanto, se necesitan esfuerzos para mejorar la sensibilidad y especificidad de las pruebas de diagnóstico, así como para aumentar la concienciación de las mujeres sobre los factores de exposición al VPH y la necesidad de la adhesión a los programas de control.

\section{Descriptores:}

Neoplasias;

\section{Papillomaviridae; Terapéutica.}

\section{Introdução}

O câncer de colo uterino é uma importante causa de morbidade e mortalidade que representa o terceiro tumor mais frequente na população feminina, atrás do câncer de mama e do 
colorretal, e a quarta causa de morte de mulheres por câncer no Brasil ${ }^{(1)}$. Por se tratar de uma causa relevante de morte por câncer, torna-se um dos maiores problemas de saúde pública do mundo principalmente nos países em desenvolvimento ${ }^{(2)}$.

A história natural do câncer cervical, tipicamente, estende-se por um período de 15 a 20 anos e envolve um número de passos críticos, incluindo infecção por papilomavírus humano (HPV), persistência da infecção, progressão para lesão precursora e finalmente desenvolvimento do câncer invasor $^{(3)}$. Segundo dados da Organização Mundial de Saúde, 99\% das lesões intraepiteliais de alto grau e dos cânceres invasivos do colo do útero são causadas pelo Papilomavírus humano (HPV) ${ }^{(4)}$.

Nos países desenvolvidos, observa-se um declínio na incidência do câncer invasor do colo uterino e uma redução de cerca de $80 \%$ da mortalidade, o que pode ser atribuído principalmente a um aumento no rastreamento de mulheres na faixa etária de risco, da detecção e do tratamento das lesões precursoras com alto potencial de malignidade ${ }^{(1)}$.

Com o esclarecimento do HPV como principal cofator para surgimento do câncer de colo uterino, a colposcopia ganhou destaque, pois é uma importante forma de detecção de lesões subclínicas do HPV e consequentemente, de avaliação da evolução dessas lesões para lesões pré-invasivas ${ }^{(5)}$.

A associação da colposcopia com a colpocitologia traz a viabilidade de estudo das lesões em colo e vagina, principalmente, com a discrição de topografia e gravidade das lesões, favorecendo dessa forma a detecção precoce de alterações pré-invasivas ${ }^{(6)}$.

Após resultado positivo para malignidade na colpocitologia ou a visualização de um colo suspeito ao exame especular, mesmo com citologia negativa, deve-se lançar mão da colposcopia, como um exame para detecção de microcarcinoma. Apesar de sofrer muitas mudanças ao longo do tempo quanto às suas indicações, técnica e instrumental, a colposcopia ao decorrer dos anos assumiu um papel bem mais amplo, não se restringindo a um simples método de orientação do local a ser realizada uma biópsia(7). Uma vez encontrada a lesão, a biópsia colposcopicamente dirigida e o estudo histopatológico são utilizados para firmar o diagnóstico, excluir doença invasiva e ajudar na decisão terapêutica.

Dentre as alternativas terapêuticas das lesões precursoras, citam-se aquelas que destroem o tecido, podendo ser 
realizadas pela diatermocoagulação, crioterapia ou com laser, e as excisionais, como a conização com bisturi ou com o laser ${ }^{(8)}$.

Distinguem-se na CAF (Cirurgia de Alta Frequência) dois tipos de procedimentos, usados de acordo com a avaliação colposcópica. A exérese da zona de transformação é indicada para pacientes com lesão ectocervical e JEC visível. A conização é a opção para pacientes que apresentam neoplasia intra-epitelial cervical acentuada (NIC III) e atipia de localização endocervical. Estas pacientes necessitam de maior ressecção do canal endocervical. A CAF vem sendo largamente utilizada para o tratamento das lesões préneoplásicas. É método de fácil e rápida execução, que pode ser realizado com anestesia local, e que possibilita o estudo histopatológico da peça operatória. Sob visão colposcópica satisfatória o procedimento pode ser realizado ambulatorialmente, permitindo o tratamento imediato das lesões - prática chamada "Ver e Tratar" 9. A estratégia dessa práticas é facilitar o acesso das mulheres ao tratamento, diminuindo a ansiedade, as possibilidades de perdas no seguimento e os custos da assistência ${ }^{(10)}$.

\section{Método}

O presente trabalho trata-se de uma pesquisa epidemiológica, transversal e retrospectiva, realizada em um Hospital Universitário da região nordeste. Foram incluídos no estudo os dados dos prontuários que se enquadravam na pesquisa através do critério de inclusão que consiste na amostra de pacientes mulheres maiores de 18 anos, atendidas no ambulatório de Patologias do Trato Genital Inferior do hospital universitário no período compreendido entre janeiro de 2012 a dezembro de 2016, que realizaramcirurgia de alta frequência sob visão colposcópicapara tratamento de lesão de alto grau de colo uterino e que apresentavam dados de citologia, colposcopia, biópsia e anatomopatológico descritas no prontuário. Foi definido como critério de exclusão do estudo prontuários e laudos de anatomopatológico de pacientes no ciclo gravídico-puerperal, portadoras da SIDA, diagnosticadas com adenocarcinoma de margens comprometidas e aquelas que não concluíram o seguimento.

Esta pesquisa foi submetida e aprovada pelo Comitê de Ética em Pesquisa da Universidade Federal de Alagoas e, posteriormente, aprovada pela Plataforma Brasil, sob parecer de número 2.482.552, seguindo os padrões 
éticos como rege a Resolução 510/16 do

Conselho Nacional de Saúde.

Para o presente estudo, não houve a necessidade de Termo de Consentimento Livre e Esclarecido (TCLE) por se tratar de uma pesquisa apenas com prontuários, na qual não houve contato com as pacientes ou exposição de dados pessoais das mesmas. A coleta de dados foi realizada no período de Março de 2018 a Abril de 2018 no Hospital Universitário do estado de Alagoas por meio do Serviço de Arquivo Médico (SAME).

Foram coletados dados de 59 prontuários, dos quais 11 não cumpriram os critérios de inclusão em virtude da ausência de resultado decolpocitologia (02), citologia (01), biópsia (03), anatomopatológico (01). Outros dois foram excluídos por abandono de seguimento, e 9 prontuários não foram encontrados no Serviço de Arquivo Médico, totalizando 18 exclusões. Assim, a amostra final foi constituída pelos dados contidos nos prontuários das 41 pacientes restantes. Nessas, observou-se dados sóciodemográficos como: idade, paridade, uso de drogas, sexarca, número de parceiros e os diagnósticos de colposcopia e de biópsia foram confrontados com os achados anatomopatológicos obtidos por $\mathrm{CAF}$ através do teste de $\mathrm{McNemar}$, avaliando a possível discordância entre eles. O banco de dados foi desenvolvido a partir do programa Microsoft Excel versão 2013.

\section{Resultados}

O estudo mostrou, conforme tabela 1, que dos 41 prontuários analisados, 29 obtiveram laudo citopatológico de lesão de alto grau, 3 de baixo grau, 2 de ASC-US, 4 de ASC-H e 3 negativos para neoplasia. Quanto ao laudo histopatológico do CAF observou-se 6 NIC II, 28 NIC III/Carcinoma in situ e 7 negativos para malignidade. Das 28 pacientes com anatomopatológico do CAF NIC 3, 2 tinham citologia ASC-US, 3 ASC-H, 1 LIE-BG e 22 LIE-AG. Em relação as 6 pacientes com NIC 2 no anatomopatológico do CAF, 4 apresentaram citologia prévia evidenciando LIE-AG, 1 ASC-H e 1 normal. Das 7 pacientes com negatividade para neoplasia no anatomopatológico, 3 obtiveram citologia prévia com resultado de LIEAG, 2 LIE-BG e 2 com ausência de neoplasia. 


\section{Tabela 1 - Resultados dos Exames Anatomopatológicos Após Cirurgia de Alta Frequência em 41 mulheres}

\begin{tabular}{lllllll}
\hline $\begin{array}{l}\text { Citopato- } \\
\text { lógico }\end{array}$ & Normal & ASCUS & ASC-H & LIEBG & LIEAG & Total \\
$\begin{array}{l}\text { Anatomo- } \\
\text { patológico }\end{array}$ & & & & & & \\
Normal & 2 & 0 & 0 & 2 & 3 & 7 \\
NIC 1 & 0 & 0 & 0 & 0 & 0 & 0 \\
NIC 2 & 1 & 0 & 1 & 0 & 4 & 6 \\
NIC 3 & 0 & 2 & 3 & 1 & 22 & 28 \\
Total & 3 & 2 & 4 & 3 & 29 & 41 \\
\hline
\end{tabular}

A caracterização das pacientes atendidas no ambulatório de Patologias do Trato Genital Inferior do hospital universitário e que realizaram cirurgia de alta frequência sob visãocolposcópica para tratamento de lesão de alto grau de colo uterino em relação à idade é apresentada na tabela
2. Observa-se no estudo a presença de pacientes com idades entre 22 e 71 anos, com predominância de mulheres com idades entre 31 e 35 anos, contabilizando $34 \%$ do universo estudado.

Tabela 2- Caracterização por Idade das Pacientes com Lesão Intraepitelial Alto Grau do Serviço de Patologias do Trato Genital Inferior

\begin{tabular}{llll}
\hline Variável & & $\mathbf{n}$ & $\mathbf{\%}$ \\
& $22-25$ anos & 3 & 7 \\
& $26-30$ & 2 & 5 \\
Idade & $31-35$ & 14 & 34 \\
& $36-40$ & 10 & 24 \\
& $41-45$ & 7 & 17 \\
& $46-50$ & 3 & 7 \\
& $>50-71$ & 2 & 5 \\
\hline
\end{tabular}

Em relação à concordância entre resultado do anatomopatológico, os achados obtidos durante a observa-se que houve uma colposcopia, quando confrontados ao correspondência entre os resultados em 
30 pacientes $(73 \%)$ e $11(27 \%)$ delas

apresentaram laudos conflitantes.

Tabela 3 - Concordância dos Resultados de Colposcopia com os Achados do Aanatomopatológico Obtido Através da Cirurgia de Alta Frequência

\begin{tabular}{ccc}
\hline Concordância & $\mathbf{n}$ & $\mathbf{\%}$ \\
Sim & 30 & 73 \\
Não & 11 & 27 \\
Total & 41 & $100 \%$ \\
\hline
\end{tabular}

Verificando-se a compatibilidade entre os resultados prévios de biópsia em relação aos achados da análise do material obtido por CAF, observa-se que em 34 dos laudos (83\%) houve relação de compatibilidade entre os resultados obtidos e em 7 resultados (17\%) houve discordância.

Tabela 4 -Concordância dos Resultados de Biópsias com os Achados no Anatomopatológico Obtido Através da Cirurgia de Alta Frequência

\begin{tabular}{ccc}
\hline Concordância & $\mathbf{N}$ & $\mathbf{\%}$ \\
Sim & 34 & 83 \\
Não & 7 & 17 \\
Total & 41 & $100 \%$ \\
\hline
\end{tabular}

Das 34 pacientes com indicação para realização de cirurgia de alta frequência sob visão colposcópica e que apresentaram $\quad 0 \quad$ laudo anatomopatológico correspondente a
LIE-AG, em 4 casos (12\%) houve necessidade de reabordagem devido a remanescência, ou recidiva, de doença local.

Tabela 5 - Pacientes Portadoras de Lesão Intraepitelial de Alto Grau que Evoluíram para Reconização no Primeiro Seguimento Pós Cirurgia de Alta Frequência

\begin{tabular}{cccc}
\hline $\begin{array}{c}\text { Necessidade de } \\
\text { Reconização }\end{array}$ & & $\mathbf{n}$ & $\mathbf{\%}$ \\
& Sim & 4 & 12 \\
& Não & 30 & 88 \\
\hline
\end{tabular}

No que concerne à obtenção de margens livres após a realização do procedimento de conização, das 41 peças estudadas, 25 (61\%) obtiveram 
margens livres, 7 (17\%) margens presença de doença comprometendo comprometidas e em 9 (22\%) delas, não margem.

havia no laudo descrição a cerca

Tabela 6 - Avaliação das Margens do Material Obtido pela Cirurgia de Alta Frequência

\begin{tabular}{llll}
\hline Variável & & $\mathbf{N}$ & $\mathbf{\%}$ \\
Margem & Livres & 25 & $61 \%$ \\
& Comprometidas & 7 & $17 \%$ \\
& Sem informação & 9 & $22 \%$ \\
& Total & 41 & $100 \%$ \\
\hline
\end{tabular}

Outra informação e caracterização do estudo dá-se em relação a história gestacional de cada paciente. Observa- se que a maioria delas, $56 \%$, apresentou mais que duas gestações prévias ao desenvolvimento da doença.

\section{Tabela 7 - Caracterização das pacientes com Lesão Intraepitelial Alto Grau com relação a número de gestações}

\begin{tabular}{cccc}
\hline Variável & & $\mathbf{n}$ & $\mathbf{\%}$ \\
Gestação & Sem gestação & 1 & $3 \%$ \\
& 1 gestação & 3 & $9 \%$ \\
& 2 gestações & 5 & $15 \%$ \\
& $>2$ gestações & 19 & $56 \%$ \\
& Sem informação & 6 & $18 \%$ \\
& Total & 34 & 100 \\
\hline
\end{tabular}

\section{Discussão}

Verifica-se neste estudo que o predomínio de mulheres portadoras de Lesão Intraepitelial de Alto Grau atendidas no serviço de ginecologia de um hospital universitário da região nordeste, encontra-se na faixa etária entre 30 e 40 anos, dado que corresponde e se associa ao encontrado na literatura, possivelmente justificado pelo período de latência de 10 a 15 anos entre a exposição ao HPV e o desenvolvimento do câncer do colo do útero $^{(11)}$.

A maioria dos fatores de risco para câncer do colo do útero está associada ao aumento do risco de contrair infecção pelo HPV (por exemplo, início precoce da atividade 
sexual, parceiros sexuais múltiplos ou de alto risco) ou capacidade reduzida de eliminar a infecção por HPV (por exemplo, imunossupressão) ${ }^{(12)}$. Dessa forma, existe uma alta prevalência de infecção por HPV em mulheres sexualmente ativas, particularmente em mulheres mais jovens. Embora uma parcela dessas mulheres eliminem a infecção pelo HPV em 8 a 24 meses $^{(13)}$, as que não eliminarem, estarão sujeitas ao período de latência para o desencadear da doença.

Entre os fatores ambientais relacionado com a infecção do HPV está a paridade. Estudos epidemiológicos têm demostrado alta associação entre a multiparidade com as lesões de alto grau e com o carcinoma cervical. A International Agency for Research on Cancer (IARC) verificou que mulheres com sete ou mais gestações a termo tiveram risco de desenvolver câncer cervical aumentado em quatro vezes mais, em comparação com as nulíparas. E 2,3 vezes mais em comparação com mulheres que tiveram uma ou duas gestações a termo ${ }^{(14)}$. No presente estudo, $56 \%$ das pacientes com lesão intra-epitelial de alto grau possuiam 3 ou mais filhos. Fato esse, que levanta o questionamento de o fenômeno gestacional ser atribuído em si como um fator de risco ou em virtude de o maior número de gestações ser decorrente de uma maior exposição a relações sexuais desprotegidas, com consequente exposição ao $\mathrm{HPV}^{(15)}$.

Em relação aos benefícios da triagem na diminuição da incidência e mortalidade do câncer do colo do útero, é preciso ser avaliado o risco de sobrediagnóstico. O rastreio com o teste de Papanicolau pode detectar anomalias que desaparecem sem intervenção, o que pode levar a procedimentos de diagnóstico e tratamento desnecessários 16. Nesse cenário, a colposcopia tem assumido um papel intermediário entre a citologia e histologia, funcionando, como um método de rastreamento de patologia cervical $^{(17)}$.

No que concerne à Cirurgia de Alta Frequência, para obtenção de uma alta sensibilidade, é preciso que o procedimento seja realizado adequadamente em todas as etapas, indo desde a correta realização do procedimento pelo profissional médico, até a interpretação das lâminas. Dessa forma, sugere-se que o valor da colposcopia sirva, sobretudo, para mais acertada localização do local a se proceder a Cirurgia de Alta Frequência, e não para aumentar a acurácia diagnóstica das lesões intraepiteliais cervicais $^{(18)}$. 
Dados da literatura mundial tem tido a preocupação dentro desse cenário, e no que tange às lesões residuais, alguns advogam que as margens livres na peça de conização não são bons preditores de ausência de doença ${ }^{(19)}$, por demonstrarem que a recorrência possa vir a estar relacionada ao HPV de alto risco e a imunossupressão, além de levar em conta a dificuldade da avaliação endocervical mesmo com o auxílio da colposcopia $^{(20)}$; Diferentemente, outros autores apontam para a margem livre como preditor de ausência de doença ${ }^{(21)}$. Na pesquisa que relatamos, apenas 9\% das doentes com margem livre apresentaram doença residual em algum seguimento. Ao contrário, cerca de $57 \%$ das doentes com margem envolvida apresentaram lesão em peça de cirurgia complementar. Os resultados descritos são ligeiramente superiores aos encontrados na literatura; no entanto, há que ter em conta o número limitado de casos.

Apesar dessa discordância, uma das grandes expectativas ao se realizar a conização do colo uterino sob visão colposcópica é a obtenção de resultados mais precisos e consequente margem livre no estudo anatomopatológico. Das 34 pacientes deste estudo com LIE-AG, 4 necessitaram da reconização no primeiro seguimento, duas tiveram margens comprometidas na primeira conização, e todas com colpocitologia, colposcopia e biopsia alteradas no seguimento.

Em virtude dos possíveis fatores associados à recidiva, indica-se melhor seguimento no primeiro ano, quando a recorrência de doença é maior ${ }^{(22)}$. Neste estudo, os seguimentos foram realizados 6 e 12 meses após o tratamento, onde foi possível observar recidiva da doença em $12 \%$ das pacientes, com consequente necessidade de reconização.

Relativamente a concordância ou discordância entre os métodos de colposcopia e biópsia em relação ao anatomopatológico, observou-se através do teste de McNemar um " $p$ " de 1 e 0,12 respectivamente, levando a conclusão de que não houve significância na discordância entre os métodos diagnósticos.

No estudo efetuado foi, ainda, de notar poucas informações quanto aos dados sócio-demográficos nos prontuários avaliados. Ressalte-se que as informações a cerca de uso de drogas, sexarca e número de parceiros das pacientes, limitam uma análise profunda quanto a essas variáveis, consequentemente acarretando limitações nas demais discussões. 


\section{Conclusão}

Os resultados encontrados nessa pesquisa, apresentam concordância com outros estudos realizados na literatura. Revisões sistemáticas e metanálises de estudos observacionais fornecem evidências consistentes e convincentes de que a triagem leva a uma diminuição na incidência e mortalidade por câncer do colo do útero, além de estar associada a maiores taxas de cura e menor risco de câncer invasivo do colo do útero.

A nossa amostra compreende-se mulheres portadoras de Lesão Intraepitelial de Alto Grau, na sua maioria entre a faixa etária de 30 e 40 anos. Depreende-se também, através dos dados expostos, alta associação entre a multiparidade com as lesões de alto grau e baixa significância na discordância entre os métodos diagnósticos avaliados.

Nomeadamente a aplicabilidade do uso da colposcopia para além de um facilitador de imagens topográficas, pode ser considerado um diferencial na abordagem da zona de transformação cervical.

Por fim, embora o câncer do colo do útero seja uma doença teoricamente passível de prevenção primária, a detecção e o tratamento adequado das lesões precursoras é atualmente a base para o controle da doença. Ainda são necessários esforços para melhorar a sensibilidade, especificidade e valores preditivos positivos e negativos dos testes diagnósticos, assim como para aumentar a conscientização das mulheres em aderirem aos programas de controle.

\section{Referências}

1. Ministério da Saúde (BR). Instituto Nacional do Câncer. Brasília: Ministério de Saúde; 2017.

2. Oliveira MM, Malta DC, Guauche H, Moura L, Silva GA. Estimativa de pessoas com diagnóstico de câncer no Brasil: dados da Pesquisa Nacional de Saúde, 2013. Rev bras epidemiol. 2015; 18(2): 146-57.

3. Frazer I. Correlating immunity with protection for HPV infection. Int $\mathrm{J}$ Infect Dis. 2007;11 Suppl 2:S10-6.

4. Spagnuolo C, Russo GL, Orhan IE, Habtemariam S, Daglia M, Sureda A, et al. Genistein and cancer: current status, challenges, and future directions. Advances in nutrition. 2015;6:408-19.

5. Lana V, Teixeira LA. A colposcopia no diagnóstico precoce do câncer do colo do útero: a experiência norte-americana. Revista Brasileira de História da Ciência. 2015; 8(1): 39-50.

6. Nascimento MI, Rabelo IMMA, Cardoso FSP, Musse RTV. Tempo de espera pela primeira colposcopia em mulheres com teste de Papanicolaou alterado. Rev. Bras. Ginecol. Obstet. 2015; 37(8):381-87. 
7. Federação

Associações

Obstetrícia

de

Brasileira

das

Orientação em Trato Genital Inferior e

Colposcopia. São Paulo: FEBRASGO; 2010.

8. Garcia Ramos AM, Garcia Ramos ES, dos Reis HLB, de Rezende RB. Quality Evaluation of Cone Biopsy Specimens Obtained by Large Loop Excision of the Transformation Zone. Journal of Clinical Medicine Research. 2015;7(4):220-24.

9. Ribeiro CM, Silva GA. Avaliação da produção de procedimentos da linha de cuidado do câncer do colo do útero no Sistema Único de Saúde do Brasil em 2015. Epidemiol. Serv. Saúde. 2018; 27(1):1-10.

10. Ebisch RM, Rovers MM, Bosgraaf RP, et al. Evidence supporting see-and-treat management of cervical intraepithelial neoplasia: a systematic review and meta-analysis. BJOG. 2016;123:59-6.

11. Frumovitz, M. Invasive Cervical Cancer: Epidemiology, Risk Factors, Clinical Manifestations, and Diagnosis. UpToDate; 2013.

12. Klumb EM, Araújo ML, Jesus GR, Santos DB, Oliveira AV, Albuquerque EM, et al. Is higher prevalence of cervical intraepithelial neoplasia in women with lupus due to immunosuppression? J Clin Rheumatol 2010; 16:1-153.

13. Ho GY, Bierman R, Beardsley L, Chang CJ, Burk RD . Natural history of cervicovaginal papillomavirus infection in young women. $\mathrm{N}$ Engl $\mathrm{J}$ Med. 1998; 338:23.
14. Muñoz N, Franceschi S, Bosetti C, Moreno V, Herrero R, Smith JS, et al. Role of parity and human papillomavirus in cervical cancer: the IARC multicentric case-control study. Lancet. 2002;359(9312):1093-01.

15. Moradi S, Tandris HM, Darvish L, Roozbeh N. Evaluating Cervicovaginal Infections and Cervical Cancer in Women with Low Socioeconomic Levels. Iranian Journal of Public Health. 2017;46(6):867-68.

16. Feldman S, Goodman A, Peipert J. Screening for cervical cancer. UpToDate; 2015.

17. Santos UM, Souza SEB. Papanicolaou: diagnóstico precoce ou prevenção do câncer cervical uterino? Rev Baiana Saúde Pública. 2013; 37(4):941-51.

18. Tavares SB, de Souza NL, Manrique EJ, Azara CZ, da Silveira EA, Amaral RG. Internal quality control for cervical cytopathology: comparison of potential false-negatives detected at rapid prescreening and at $100 \%$ rapid review. Acta Cytol. 2014;58:439-45.

19. Hiramatsu K, Ueda Y, Yoshino K, Fujita M, Morii E, Enomoto T, Kimura $T$. Conization using the Shimodarira.Taniguchi procedure for adenocarcinoma in situ of the uterine cérvix. Eur J Obstet Gynecol Reprod Biol. 2013;168:218-21.

20. Tavares MV, Gouveia A. Adenocarcinoma in situ do colo do útero: experiência de um centro de referência. Acta Obstet Ginecol Port. 2016; 10(2): 96-01. 
21. Costales AP, Milbourne AM, Rhodes HE, Munsell MF, Wallbillich JJ, Brown J, et al. Risk of residual disease and invasive carcinoma in women treated for adenocarcinoma in situ of the cervix. Gynecol Oncol. 2013;129:513-16.

22. Araújo CFL. Neoplasia intraepitelial cervical: envolvimento das margens de ressecção, após excisão eletrocirúrgica com alça e recidiva da doença. [Monografia de conclusão de curso de medicina] da Universidade Federal da Bahia. Salvador; 2013. Disponível em: https://repositorio.ufba.br/ri/handle/ri/1 4248. 